\title{
Comparative Analysis of Lactulose and Fructooligosaccharide on Growth Kinetics, Fermentation, and Antioxidant Activity of Common Probiotics
}

\author{
Evelyn Lu1, Marie Yeung1, Chi Kong Yeung2* \\ ${ }^{1}$ Biological Sciences Department, California Polytechnic State University, San Luis Obispo, CA, USA \\ ${ }^{2}$ Animal Science Department, California Polytechnic State University, San Luis Obispo, CA, USA \\ Email: *ckyeung@calpoly.edu
}

How to cite this paper: Lu, E., Yeung, M. and Yeung, C.K. (2018) Comparative Analysis of Lactulose and Fructooligosaccharide on Growth Kinetics, Fermentation, and Antioxidant Activity of Common Probiotics. Food and Nutrition Sciences, 9, 161-178. https://doi.org/10.4236/fns.2018.93013

Received: January 31, 2018

Accepted: March 2, 2018

Published: March 5, 2018

Copyright $\odot 2018$ by authors and Scientific Research Publishing Inc. This work is licensed under the Creative Commons Attribution International License (CC BY 4.0).

http://creativecommons.org/licenses/by/4.0/

cc) (i) Open Access

\begin{abstract}
Prebiotics are non-digestible oligosaccharides that selectively stimulate the growth of beneficial bacteria in the human gut. Fructooligosaccharide (FOS) is a common prebiotic found in food products and infant formula. Lactulose is primarily used as a pharmaceutical ingredient but also shows potential prebiotic activities. Our objectives were to determine and compare the effects of FOS and lactulose on: 1) growth kinetics of common probiotics in aerobic condition; 2) $\mathrm{pH}$ and titratable acidity after fermentation; and 3) antioxidant capacity of the probiotics. Ten probiotic and two non-probiotic strains, representing genera Lactobacillus, Bifidobacterium, Bacillus, and Escherichia were assembled. Media used for prebiotics experiment were modified to contain $2 \%$ FOS or lactulose as the sole or main carbohydrate source. All experiments were done in triplicate. In aerobic condition, most strains cultured with FOS or lactulose did not grow optimally compared to dextrose (a non-prebiotic), while all four Bifidobacterium spp. showed little growth regardless of the carbohydrate source. In anaerobic condition, lactulose and FOS fermentation of Bifidobacterium spp. yielded similar $\mathrm{pH}(p=0.2723)$, but percent lactic acid, as determined by titratable acidity, was higher after lactulose fermentation $(p=$ 0.0004). The non-probiotic strains were able to utilize both FOS and lactulose, but displayed weaker acid production and higher $\mathrm{pH}(p<0.0001)$ relative to the probiotic strains. Antioxidant activity of spent medium was measured with Trolox as the reference standard. Overall, the antioxidant activity of probiotics was strain-dependent. FOS enhanced the antioxidant activity of Bifidobacterium spp. ( $p=0.0002)$ and Lactobacillus spp. $(p=0.0447)$, but not probiotic $E$. coli and Bacillus spp. $(p=0.2599)$ or non-probiotics $(p=0.8816)$.
\end{abstract}


In conclusion, lactulose supported growth activities of probiotics to a similar extent as FOS. Lactulose also stimulated higher acid production for Bifidobacterium spp. than FOS in anaerobic condition, thus it might be considered for incorporation into functional food products containing bifidobacteria.

\section{Keywords}

Prebiotics, Oligosaccharides, Lactulose, FOS, Probiotics

\section{Introduction}

The gut microbes play an important role in human health. Soon after birth, microbes immediately colonize human skin, oral cavity, and gastrointestinal tract surfaces [1]. A diverse and large amount of bacteria populate the human gastrointestinal tract forming a complex ecosystem of intestinal microbiota [2]. These microbes are an integral part of the gastrointestinal tract due to their role in conserving gut homeostasis, where gut microbes and the human host work together to create a stable ecosystem that provides appropriate responses to $\mathrm{mi}$ crobial "friend" or "foe" [3].

Non-digestible oligosaccharides such as fructooligosaccharide (FOS) and galactooligosaccharide (GOS) are considered prebiotics, which according to Gibson et al. (2010), are defined as selectively fermented ingredients that result in specific changes, in the composition and/or activity of the gastrointestinal microbiota, thus conferring benefits upon host health [4]. Some reported benefits include enhanced immune system [5] and defense against pathogens [6], modulation of short-chain fatty acid (SCFA) production [7] and tight junction integrity [8], and mild improvement in iron status [9]. Proliferation of beneficial bacteria, usually lactobacilli and bifidobacteria, in the colon of the host may also be associated with reducing lipid peroxidation in colonic mucosa of intact mammals [10] [11]. The putative antioxidant effect might be due to the ability of the bacteria to scavenge free radicals, and/or an increase in antioxidant capacities of the colon contents. Oxidative stress in the colonic mucosa is presumably involved in the pathogenesis of colon cancer [12].

Lactulose is derived from lactose through an isomerization process in which the glucose moiety in the lactose molecule is converted to fructose, resulting in a disaccharide of galactose and fructose linked together via a $\beta 1 \rightarrow 4$ glycosidic linkage [4] [13]. Lactulose has been shown to increase the viability of Bifidobacterium and Lactobacillus spp. both in vitro and in vivo [14] [15] [16], and is therefore conventionally classified as a prebiotic [17]. Although available as a functional food ingredient, lactulose is primarily used as a pharmaceutical [18]. On the other hand, FOS is derived from plant sources such as chicory roots [19] [20], and structurally it is a linear chain of $\beta$-fructan with the number of fructose unit typically ranging from 2 - 10 [4]. FOS is considered an important commercial prebiotic [21], and studies have examined its use as a functional food ingre- 
dient under different food processing environments [22] [23].

The objectives of this study were to determine the prebiotic effects of lactulose on the growth, fermentative ability, and antioxidant activity of common probiotics. The effects of FOS and dextrose were also determined to allow comparisons so as to achieve a better characterization of the prebiotic potential of lactulose as a functional food ingredient.

\section{Materials and Methods}

\subsection{Bacterial Strains}

In order to compare the effects of lactulose and FOS on the growth of beneficial bacteria, ten probiotic and two non-probiotic strains, representing genera Lactobacillus, Bifidobacterium, Bacillus, and Escherichia were assembled (Table 1). Six species, Lactobacillus casei, Lactobacillus acidophilus, Bifidobacterium bifidum, Bifidobacterium animalis, Escherichia coli and Bacillus pumilus, were purchased from Hardy Diagnostics (Santa Maria, CA). The Lactobacillus and Bifidobacterium spp. are considered probiotic-type bacteria, whereas $E$. coli and $B$. pumilus are non-probiotics. In addition, several more probiotics were isolated from commercial products. The species identities according to the product labels were Lactobacillus rhamnosus, L. casei, Bifidobacterium infantis, Bifidobacterium lactis, and Bacillus coagulans. Lactobacillus rhamnosus (LGG) was isolated from the product by incubating a supplement tablet in De Man, Rogosa, Sharpe broth (MRS broth; Hardy Diagnostics, Santa Maria, CA), whereas L. casei LcS was isolated by inoculating MRS broth with an aliquot of a probiotic drink obtained from a local grocery store, followed by a $24-\mathrm{hr}$ anaerobic incubation at

Table 1. Bacterial strains used in the study and their sources. Identities of strains that were isolated from commercial products were based on information found on product labels.

\begin{tabular}{cc}
\hline Bacterial strain & Source \\
\hline Lactobacillus acidophilus ATCC 4356 & Hardy Diagnostics \\
Lactobacillus casei ATCC 334 & Hardy Diagnostics \\
Lactobacillus rhamnosus GG (LGG) ATCC 53103 & Commercial probiotic supplement \\
Lactobacillus casei & Commercial probiotic drink \\
(a proprietary strain, designated as LcS in this study) & Hardy Diagnostics \\
Bifidobacterium bifidum ATCC 11863 & Hardy Diagnostics \\
Bifidobacterium animalis ssp. animalis ATCC 25527 & Commercial probiotic supplement \\
Bifidobacterium infantis 35624 & Commercial yogurt \\
Bifidobacterium lactis DN173-010 & University of Rhode Island \\
Escherichia coli Nissle 1917 (ECN) & (Dr. Paul S. Cohen) \\
Bacillus coagulans GBI-30 & Commercial probiotic supplement \\
Escherichia coli (JM 101) ATCC 33876 & Hardy Diagnostics \\
Bacillus pumilus ATCC 14884 & Hardy Diagnostics \\
\hline
\end{tabular}


$37^{\circ} \mathrm{C}$ in a GasPak jar (Becton, Dickinson and Company, Franklin Lakes, NJ). The overnight cultures were streaked for isolation on MRS agar and incubated at $37^{\circ} \mathrm{C}$ in anaerobic condition for $48 \mathrm{hr}$. Bif. infantis and Bif. lactis were isolated by growing the commercial products using Reinforced Clostridium Medium (RCM; Hardy Diagnostics, Santa Maria, CA) for $24 \mathrm{hrs}$ at $37^{\circ} \mathrm{C}$ in anaerobic condition. Similarly, the overnight cultures were streaked on RCM agar and the plates were incubated anaerobically at $37^{\circ} \mathrm{C}$ for $48 \mathrm{hr}$. B. coagulans culture was isolated by inoculating the product into Tryptic Soy Broth (TSB; Becton, Dickinson and Company, Franklin Lakes, NJ) continued with aerobic incubation for $24 \mathrm{hr}$ at $37^{\circ} \mathrm{C}$. The broth culture was streaked on a Tryptic Soy Agar (TSA) medium and incubated for $48 \mathrm{hr}$ aerobically at $37^{\circ} \mathrm{C}$. One more strain, $E$. coli Nissle 1917 (ECN), was obtained from University of Rhode Island (Dr. Paul S. Cohen). Unlike the aforementioned E. coli strain, this ECN strain is considered a probiotic strain [24] [25]. All bacterial strains were checked for purity in their respective agar medium via Gram stain.

\subsection{Culture Conditions}

All Lactobacillus strains were maintained in MRS agar medium supplemented with $0.2 \%(\mathrm{w} / \mathrm{v})$ sodium thioglycollate (Becton, Dickinson and Company, Franklin Lakes, NJ). Bifidobacterium strains were maintained in RCM agar medium supplemented with $0.2 \%$ sodium thioglycollate. Both Lactobacillus and Bifidobacterium strains were cultured at $37^{\circ} \mathrm{C}$ in an anaerobic GasPak jar with an AnaeroPack ${ }^{\circledast}$ system (Mitsubishi Gas Chemical Company, New York, NY) for 16 - $24 \mathrm{hr}$ prior to experiments. E. coli strains and Bacillus species were prepared for experiments by growing culture in TSB followed by overnight incubation (16 - $24 \mathrm{hr}$ ) at $37^{\circ} \mathrm{C}$ aerobically. To make frozen stocks, cultures of all bacterial strains were prepared in their corresponding growth medium supplemented with $20 \%$ glycerol (Sigma Aldrich, St. Louis, MO) and stored in $-80^{\circ} \mathrm{C}$ freezer.

\subsection{Growth Substrates}

FOS (Sigma Aldrich, St. Louis, MO), lactulose (Alfa Aesar, Haverhill, MA) and dextrose (Hardy Diagnostics, Santa Maria, CA) were prepared by dissolving the powder in deionized water to yield a $10 \%(\mathrm{w} / \mathrm{v})$ stock solution. The $10 \%$ stock solution was filter sterilized and stored at room temperature. An appropriate amount of either FOS or lactulose stock solution was then added to the tempered MRS basal medium (i.e., MRS without carbohydrate) to yield modified MRS with $2 \%$ prebiotics as the sole carbohydrate. The control (non-prebiotic), which was regular MRS medium containing 2\% dextrose, was prepared using premixed MRS powder. The RCM medium was prepared by adding $1.5 \%$ dextrose to the premixed powder solution that already contained $0.5 \%$ basal dextrose to yield a final concentration of $2 \%$ dextrose. For the prebiotics experiments on Bifidobacterium spp., $1.5 \%$ of either FOS or lactulose was added instead of dextrose. For experiments on E. coli and Bacillus spp., the basal medium of peptone broth (without carbohydrate) was first prepared and autoclaved, fol- 
lowed by adding an appropriate amount of the stock solution of dextrose, FOS, or lactulose to the tempered broth to reach a final concentration of $2 \%$.

\subsection{Growth Kinetics}

Growth curves were determined on bacterial cultures of the ten probiotic and the two non-probiotic strains grown overnight in their respective basal medium with $2 \%$ dextrose, FOS, or lactulose as the primary carbohydrate source. Lactobacillus, E. coli and Bacillus spp. were grown in a single carbohydrate source, whereas Bifidobacterium spp. were grown in either $2 \%$ dextrose, $0.5 \%$ dextrose plus $1.5 \%$ FOS, or $0.5 \%$ dextrose plus $1.5 \%$ lactulose. For each bacterial strain, 2 $\mu \mathrm{L}$ of overnight culture was inoculated in duplicate into $200 \mu \mathrm{L}$ of their respective medium in a 96-well plate. The plate was then incubated at $37^{\circ} \mathrm{C}$ for 24 hours in SpectraMax Plus 384 Microplate Reader (Molecular Devices, Sunnyvale, $\mathrm{CA}$ ) where the start and final $\mathrm{OD}_{600}$ of bacterial growth in aerobic condition was measured. $\mathrm{OD}_{600}$ of the culture medium alone was used as the blank. The $\Delta \mathrm{OD}_{600}$ of the sample was calculated with the following equation:

$$
\begin{aligned}
\Delta \mathrm{OD}_{600}= & (\text { sample final OD }- \text { sample start OD }) \\
& -(\text { blank final OD }- \text { blank start OD })
\end{aligned}
$$

\subsection{Fermentation Assay}

Similar to the growth curve experiments, overnight cultures of the Lactobacillus, Bifidobacterium, E. coli and Bacillus spp. were grown in their respective dextrose-, FOS-, or lactulose-containing medium. Instead of a 96-well plate, broth media were added into sterile glass test tubes containing a Durham tube which collects gas products as a result of fermentation. Overnight cultures were used to inoculate the $10 \mathrm{~mL}$-broth in the test tube at $10 \%$ inoculum level. All tubes were incubated overnight at $37^{\circ} \mathrm{C}$ in anaerobic condition. At the end of the incubation period, the tubes were centrifuged at $2,000 \times \mathrm{g}$ for $>2$ minutes to obtain a cell pellet in the bottom. Supernatant was collected and $\mathrm{pH}$ was measured using Orion 3 Star $\mathrm{pH}$ meter (Thermo Scientific, Waltham, MA). The blank culture medium was the negative control. In addition to $\mathrm{pH}$, acid production due to fermentation was also measured by titratable acidity (expressed as \% lactic acid), in which the supernatant was titrated with $0.1 \mathrm{~N} \mathrm{NaOH}$.

$$
\% \text { lactic acid }=\frac{(0.1 \mathrm{~N} \mathrm{NaOH})(\text { Volume of } \mathrm{NaOH} \text { used })(9)}{\text { Volume of sample }}
$$

The final \% lactic acid was calculated by subtracting the \% lactic acid of the blank medium from that of the sample.

\subsection{Antioxidant Activity after Fermentation}

Antioxidant activity after fermentation of prebiotics by the bacterial strains was measured using Antioxidant Assay Kit CS0790 (Sigma Aldrich, St. Louis, MO) according to the manufacturer's procedure. Briefly, overnight cultures of the 
bacterial strains were prepared in their respective medium with dextrose, FOS, or lactulose. Approximately $10^{6}$ cells were collected and mixed with $0.5 \mathrm{~mL}$ of cold 1X Assay Buffer (Sigma Aldrich, St. Louis, MO), then centrifuged at 2,000 $\times$ $\mathrm{g}$ for 2 minutes (Benchmark Scientific, Edison, NJ). The samples were kept on ice while the cell pellets were sonicated at $22.5 \mathrm{kHz}$ for 10 seconds (Thermo Fisher Scientific, Waltham, MA). The samples were then centrifuged again at $12,000 \times \mathrm{g}$ for 15 minutes at $4^{\circ} \mathrm{C}$ (GMI Inc., Ramsey, MN). Supernatants were collected and the Trolox equivalent antioxidant capacity (TEAC) was measured. Trolox is a water-soluble vitamin $\mathrm{E}$ analog that served as a control antioxidant.

Various Trolox Standards (Sigma Aldrich, St. Louis, MO) were prepared to reach final Trolox concentrations of $0 \mathrm{mM}, 0.07 \mathrm{mM}, 0.28 \mathrm{mM}, 0.50 \mathrm{mM}, 0.70$ $\mathrm{mM}, 0.80 \mathrm{mM}, 0.90 \mathrm{mM}$ and $1.00 \mathrm{mM}$. The Trolox Standards were used to construct a Trolox standard curve to calculate the TEAC levels of the samples. In a 96-well plate, $10 \mu \mathrm{L}$ each of the Trolox Standards and cell-free supernatant samples were added in duplicate, followed by additions of $20 \mu \mathrm{L}$ of Myoglobin Working Solution (Sigma Aldrich, St. Louis, MO) and $150 \mu \mathrm{L}$ of ABTS (2,2'-azino-bis (3-ethylbenzthiazoline-6-sulfonic acid)) Substrate Working Solution (Sigma Aldrich, St. Louis, MO) to each well, continued by a 5-minute incubation at room temperature. Each well was mixed with $100 \mu \mathrm{L}$ of Stop Solution (Sigma Aldrich, St. Louis, MO) at room temperature to stop the reaction. The endpoint absorbance was read at $405 \mathrm{~nm}$ via SpectraMax Plus 384 Microplate Reader (Molecular Devices, Sunnyvale, CA).

The average absorbance values from the Trolox Standards at different concentrations were used to plot a standard curve to obtain a linear regression equation (and thus the intercept and slope). The TEAC was calculated with the following equation:

$$
\operatorname{TEAC}(\mathrm{mM})=\frac{(\text { Average absorbance of test sample at } 405 \mathrm{~nm})-\text { Intercept }}{\text { Slope }}
$$

The final TEAC (mM) was calculated by subtracting the TEAC of the blank medium from that of the sample.

\subsection{Statistical Analysis}

Each experiment was performed independently at least three times. Data sets were checked for normality, and transformed if necessary, before they were analyzed using a general linear model to determine the effects of bacterial species and carbohydrate source. Pairwise comparison among treatments was done by Tukey's HSD with a Bonferroni correction. All statistical analyses were performed with JMP (version 12.1.0, SAS Institute Inc.) with statistical significance indicated by $p$-values of $<0.05$.

\section{Results and Discussion}

\subsection{Bacterial Growth}

The aerobic growth activities at $37^{\circ} \mathrm{C}$ of the ten probiotics and two non-probiotics 
were examined for 24 hours in medium containing dextrose (control), FOS, or lactulose as the main carbohydrate source. The amount of growth was expressed as $\triangle \mathrm{OD}_{600}$. When all species were considered, there was a significant decrease in $\Delta \mathrm{OD}_{600}$ when the medium was supplemented with FOS $(p=0.0098)$ in comparison to either dextrose or lactulose. $\Delta \mathrm{OD}_{600}$ of Lactobacillus spp. was significantly affected by the carbohydrate source (Table 2). LGG grew significantly better in dextrose than in either FOS or lactulose $(p<0.0001)$. A similar trend was observed in $L$. acidophilus $(p=0.0027)$. On the other hand, L. casei LcS did not grow as well in medium with FOS than in dextrose or lactulose ( $p=0.0047) . L$. casei ATCC 334 was the only Lactobacillus strain tested that did not exhibit a significantly different aerobic growth pattern in any of the carbohydrate sources provided (Figure 1). Previous studies indicated that L. acidophilus ATCC 4356 and LGG performed better with medium supplemented with dextrose than with

Table 2. Mean $\pm \mathrm{SE}$ of $\Delta \mathrm{OD}_{600}$ for the four bacterial groups with different carbohydrate sources. Cultures were grown aerobically for $24 \mathrm{hrs}$ at $37^{\circ} \mathrm{C}$. Each of the Lactobacillus spp. and Bifidobacterium spp. groups included four species/strains; the non-lactic probiotics group included $E$. coli Nissle 1917 and B. coagulans; the non-probiotics group included E. coli and B. pumilus.

\begin{tabular}{ccccc}
\hline \multicolumn{4}{c}{$\Delta \mathrm{OD}_{600}$} \\
\hline & Lactobacillus spp. & Bifidobacterium spp. Non-lactic probiotics & Non-probiotics \\
\hline Dextrose & $1.54 \pm 0.13^{\mathrm{a}}$ & $0.14 \pm 0.04^{\mathrm{c}}$ & $1.00 \pm 0.15^{\mathrm{ab}}$ & $0.77 \pm 0.11^{\mathrm{b}}$ \\
FOS & $0.94 \pm 0.20^{\mathrm{b}}$ & $0.22 \pm 0.09^{\mathrm{c}}$ & $0.77 \pm 0.08^{\mathrm{ab}}$ & $0.99 \pm 0.11^{\mathrm{ab}}$ \\
Lactulose & $1.14 \pm 0.12^{\mathrm{ab}}$ & $0.18 \pm 0.03^{\mathrm{c}}$ & $0.90 \pm 0.08^{\mathrm{ab}}$ & $1.12 \pm 0.09^{\mathrm{ab}}$ \\
\hline
\end{tabular}

$\overline{\mathrm{a}, \mathrm{b}, \mathrm{c}}$ Means with different superscripts are significantly different per Tukey's HSD with a Bonferroni correction $(p<0.05)$.

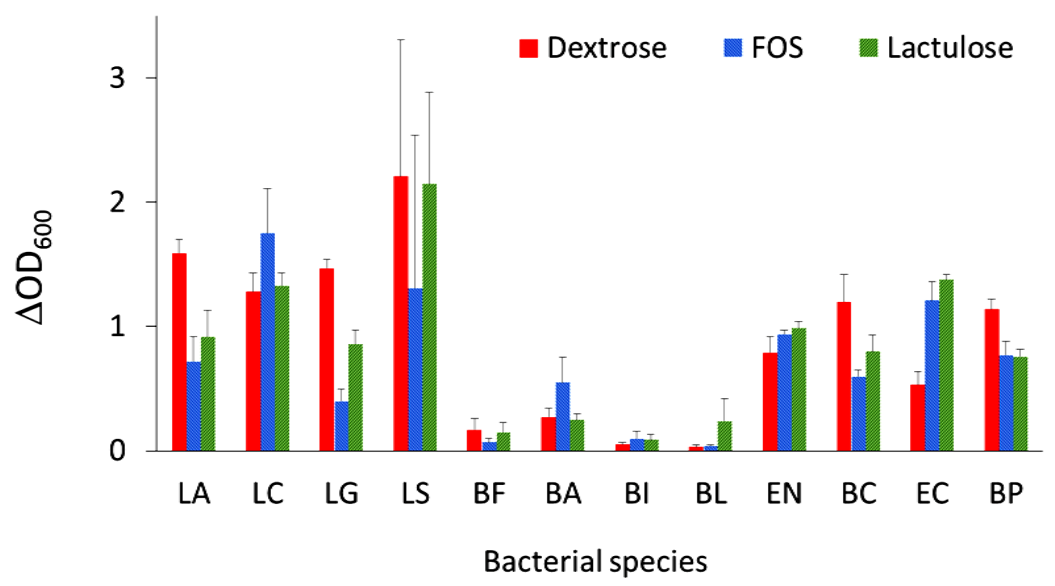

Figure 1. Mean $\pm \mathrm{SE}$ of $\Delta \mathrm{OD}_{600}$ for each bacterial species/strain grown in different carbohydrate sources following aerobic incubation at $37^{\circ} \mathrm{C}$ for $24 \mathrm{hrs}$. Error bars indicate standard error of mean. $\mathrm{LA}=$ L. acidophilus, $\mathrm{LC}=$ L. casei $\mathrm{ATCC} 334, \mathrm{LG}=$ L. rhamnosus $\mathrm{GG}, \mathrm{LS}=L$. casei $\mathrm{LcS}, \mathrm{BF}=$ Bif. bifidum, $\mathrm{BA}=$ Bif. animalis, $\mathrm{BI}=$ Bif. infantis, $\mathrm{BL}=$ Bif. lactis, $\mathrm{EN}=E$. coli Nissle, $\mathrm{BC}=B$. coagulans, $\mathrm{EC}=E$. coli $($ non-probiotic), $\mathrm{BP}=B$. pumilus (non-probiotic). 
lactulose, FOS, or inulin. Similarly, L. casei DN-144-001 did not perform as well when utilizing FOS or inulin as opposed to dextrose [26] [27]. Our results showed that the four Lactobacillus strains used in this study generally grew better aerobically when dextrose was the sole carbohydrate source. Dextrose, when available, is the preferred carbohydrate source likely because it is transported into the bacterial cytosol through conservative uptake pathways [28]. Compared to Lactobacillus, Bifidobacterium spp. grew poorly in aerobic condition regardless of the carbohydrate source (Table 2). It is not unexpected because bifidobacteria primarily have been considered obligate anaerobes [29].

While historically most probiotics are Gram-positive non-sporeforming bacteria such as those in the genus Lactobacillus or Bifidobacterium, some commercially available probiotics are Gram-negative or spore-forming. E. coli Nissle 1917 (ECN) could be considered a probiotic because it had been shown to maintain remission in ulcerative colitis patients, reduce relapse occurrence in Crohn's disease patients, and alleviate acute diarrheal symptoms in children [30] [31] [32]. Another non-lactic probiotic, B. coagulans, was reported to have beneficial effects on reducing urinary tract infections [33], preventing antibiotic-associated diarrhea in children [33], and relieving symptoms of rheumatoid arthritis [34]. B. coagulans started gaining popularity as a probiotic in recent years due to its ability to form endospores, which are resistant to high heat (a common food processing treatment) and acidity of the stomach. As a result, the number of viable cells (spores) reaching the human gut is increased, thereby delivering the putative health benefits [34] [35].

The effects of FOS and lactulose on the growth of ECN and B. coagulans were therefore examined. As a group, these non-lactic probiotics did not yield significant changes in $\Delta \mathrm{OD}_{600}$ among the different types of carbohydrate sources $(p=$ 0.7172 ). They generally grew sufficiently with any carbohydrate source in aerobic condition, except for the reduced growth observed in B. coagulans with FOS. These results are consistent with previous studies that showed some pathogenic and commensal E. coli strains could metabolize FOS [36] [37] [38]. Interestingly, while there were no statistically significant differences in $\Delta \mathrm{OD}_{600}$ among the carbohydrate sources for ECN, the $\Delta \mathrm{OD}_{600}$ of non-probiotic E. coli ATCC 33876 was significantly lower in dextrose than in FOS or lactulose $(\mathrm{p}<0.0001$, Figure 1). B. pumilus, another sporeforming Bacillus spp yet non-probiotic, exhibited similar growth as $B$. coagulans with all substrates.

\section{2. $\mathrm{pH}$ and Lactic Acid Production}

Fermentative activities of the probiotic and non-probiotic strains in anaerobic condition were evaluated by measuring $\mathrm{pH}$ and titratable acidity (expressed as \% lactic acid) post-incubation. Except for the group containing Bifidobacterium spp., fermentation using dextrose as the sole carbohydrate source resulted in overall lowest $\mathrm{pH}$, followed by lactulose and FOS ( $p<0.0001$, Table 3$)$. A potential benefit of prebiotics and probiotics consumption to the host is their ability to 
Table 3. Mean \pm SE of $\mathrm{pH}$ of the supernatant for the four bacterial groups with different carbohydrate sources after anaerobic incubation for $24 \mathrm{hrs}$ at $37^{\circ} \mathrm{C}$. Each of the Lactobacillus spp. and Bifidobacterium spp. groups included four species/strains; the non-lactic probiotics group included E. coli Nissle 1917 and B. coagulans; the non-probiotics group included E. coli and B. pumilus.

\begin{tabular}{ccccc}
\hline \multicolumn{4}{c}{$\mathrm{pH}$} \\
\hline & Lactobacillus spp. & Bifidobacterium spp. Non-lactic probiotics & Non-probiotics \\
\hline Dextrose & $3.92 \pm 0.10^{\mathrm{f}}$ & $5.01 \pm 0.21^{\mathrm{bcde}}$ & $3.96 \pm 0.05^{\mathrm{ef}}$ & $5.08 \pm 0.14^{\mathrm{bcde}}$ \\
FOS & $5.38 \pm 0.21^{\mathrm{bc}}$ & $4.88 \pm 0.20^{\mathrm{bcdef}}$ & $5.64 \pm 0.29^{\mathrm{abcd}}$ & $6.27 \pm 0.10^{\mathrm{a}}$ \\
Lactulose & $5.02 \pm 0.17^{\text {cde }}$ & $4.37 \pm 0.19^{\text {def }}$ & $4.10 \pm 0.15^{\text {cdef }}$ & $5.91 \pm 0.12^{\mathrm{ab}}$ \\
\hline
\end{tabular}

a,b,c,d,e,f Means with different superscripts are significantly different per Tukey's HSD with a Bonferroni correction $(p<0.05)$.

decrease gut $\mathrm{pH}$ which could suppress pathogens and thereby improve host resistance against intestinal disturbances [39]. As shown in Figure 2, the Lactobacillus species/strains produced more acid and lower $\mathrm{pH}$ when dextrose was the sole carbohydrate source, when compared to FOS and lactulose $(p<0.0001)$. The only exception was fermentation by $L$. casei ATCC 334 which yielded similar $\mathrm{pH}$ regardless of the carbohydrate source (Figure 2(a)). Although there tends to be an inverse relationship between $\mathrm{pH}$ and acid concentration, the amount of organic acids produced by the bacteria cannot be directly measured by $\mathrm{pH}$ alone. Since lactic acid is the primary organic acid produced by most lactobacilli, \% lactic acid in the medium post-fermentation was measured in the present study. As a group, dextrose fermentation by lactobacilli resulted in higher \% lactic acid compared to FOS $(p<0.0001)$ or lactulose $(p=0.0021$, Figure $2(b))$ fermentation. Between the two prebiotic supplements, lactulose appeared to increase \% lactic acid more than FOS. The most pronounced difference could be seen in $L$. casei LcS $(p<0.0001$, Figure 2(b)).

In a screening study on acid production by Lactobacillus spp., Kaplan and Hutkins (2000) showed that 12 of 16 strains tested (including four L. acidophilus strains and two L. casei strains) induced a color change in MRS agar medium supplemented with $2 \%$ FOS and a $\mathrm{pH}$ indicator bromcresol [27], indicating the strains' ability to ferment FOS. Our results corroborated their findings in which all four Lactobacillus strains tested in this study could ferment FOS and lactulose, although dextrose fermentation yielded lower $\mathrm{pH}$ and higher $\%$ lactic acid.

For the group of bifidobacteria containing four species, while all three carbohydrate sources were fermented to produce acid and hence lowering the $\mathrm{pH}$, lactulose supplement in the medium facilitated higher lactic acid production ( $p$ $<0.0001)$ and lower $\mathrm{pH}(p=0.0122)$ than dextrose alone (Figure 3$)$. Lactulose fermentation by Bifidobacterium spp. produced a significantly higher \% lactic acid compared to FOS fermentation ( $p=0.0004$; Figure $3(\mathrm{~b})$ ). The difference in \% lactic acid production between lactulose and FOS could not be derived from $\mathrm{pH}$ values which were statistically insignificant $(p=0.2723$; Figure 3(a)). 


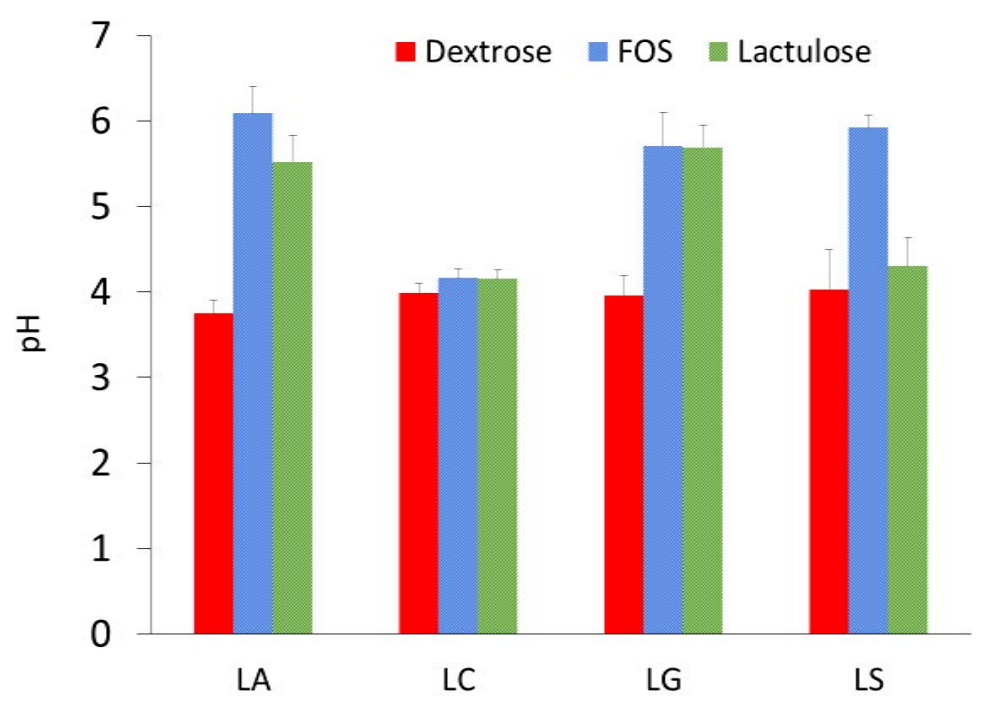

(a)

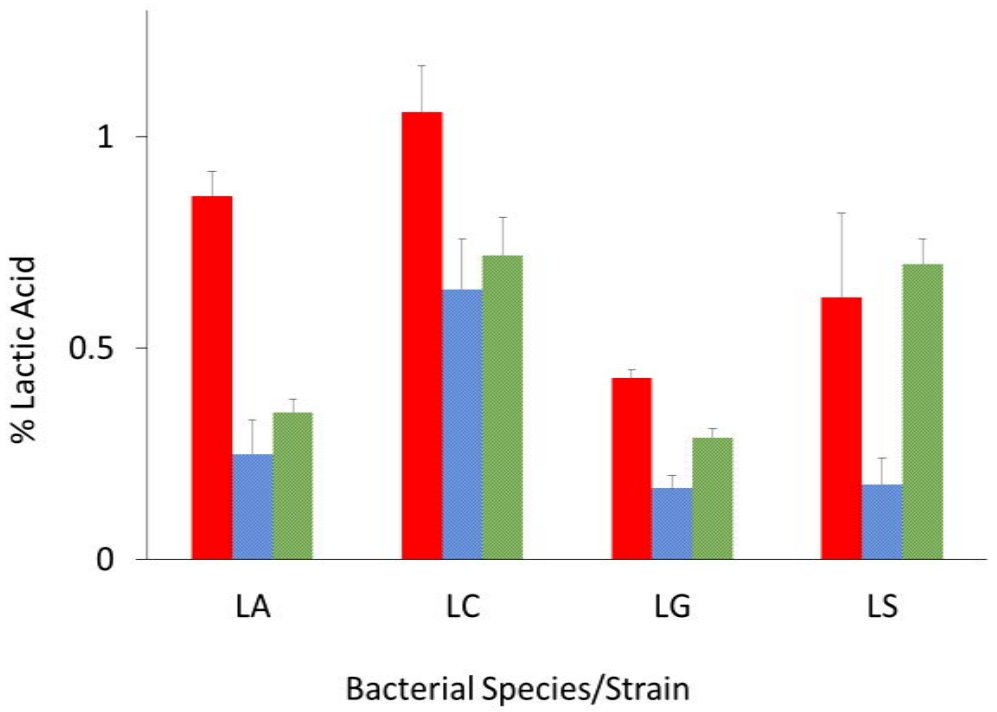

(b)

Figure 2. Mean $\pm \mathrm{SE}$ of $\mathrm{pH}$ (a) and \% lactic acid (b) of individual Lactobacillus spp. after anaerobic incubation for $24 \mathrm{hrs}$ at $37^{\circ} \mathrm{C}$ in medium containing dextrose, FOS, or lactulose as the sole carbohydrate source. Error bars indicate standard error of mean. LA = L. acidophilus, $\mathrm{LC}=$ L. casei $\mathrm{ATCC} 334, \mathrm{LG}=$ L. rhamnosus $\mathrm{GG}, \mathrm{LS}=$ L. casei $\mathrm{LcS}$.

These results further highlight the importance of measuring weak acid content by titration in addition to $\mathrm{pH}$.

Bifidobacteria are important beneficial intestinal microorganisms in the human intestinal environment. They are predominant in the gut of full-term breast-fed infants, presumably offering protection against potential pathogens. Other obligate anaerobes such as Clostridium spp. are rarely isolated in such environment [40]. Bifidobacterium spp. isolated from infant stools have been shown to exert antimicrobial effects on Listeria monocytogenes and Salmonella Typhimurium [41]. The environmental condition in vivo (i.e., human colon) 


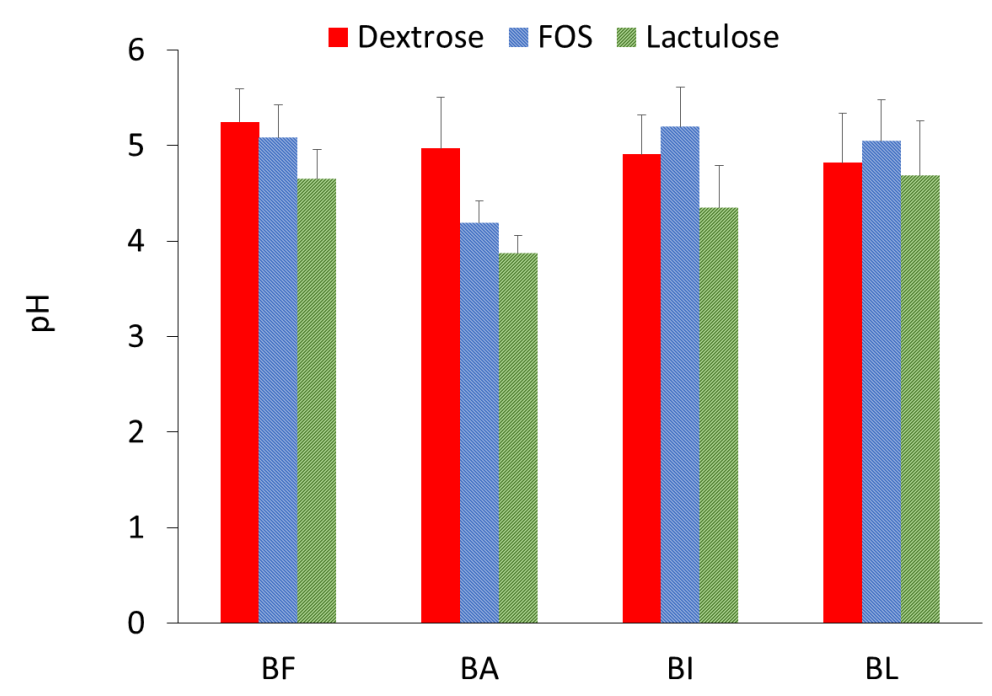

(a)

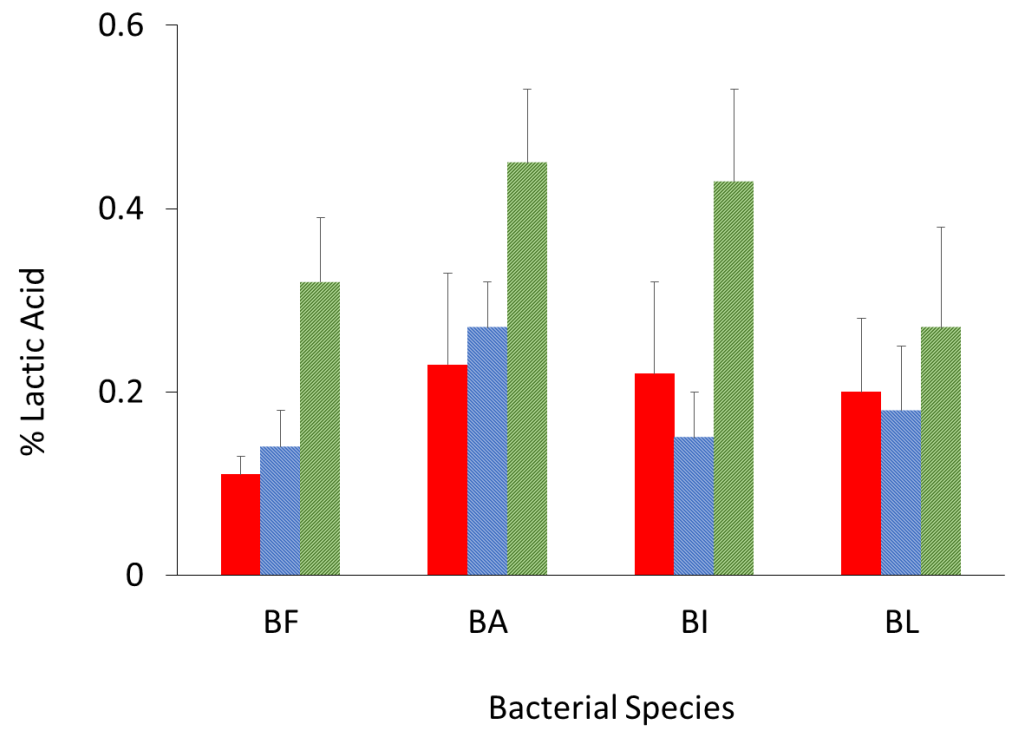

(b)

Figure 3. Mean $\pm \mathrm{SE}$ of $\mathrm{pH}$ (a) and \% lactic acid (b) of each Bifidobacterium spp. after anaerobic incubation for $24 \mathrm{hrs}$ at $37^{\circ} \mathrm{C}$ in medium containing dextrose, FOS, or lactulose as the primary carbohydrate source. Error bars indicate standard error of mean. $\mathrm{BF}=$ Bif. bifidum, $\mathrm{BA}=$ Bif. animalis, $\mathrm{BI}=$ Bif. infantis, $\mathrm{BL}=$ Bif. lactis.

where bifidobacteria most likely carry out fermentation of carbohydrates is anaerobic. Previous studies showed that more than fifty Bifidobacterium strains were found to ferment FOS [42] [43]. Langlands et al. (2004), upon examination of biopsy samples taken from the colon and rectum of human subjects during colonoscopy, confirmed that dietary FOS supplementation led to increases in surface counts of Bifidobacterium spp. [44]. Our results not only showed that different Bifidobacterium spp were able to ferment FOS, but lactulose could be an even more effective prebiotic than FOS in stimulating the fermentative ability of Bifidobacterium spp. in anaerobic condition. 
Between the non-lactic probiotics ECN and B. coagulans, the former species produced higher \% lactic acid when dextrose was the sole carbohydrate source $(p=0.0123)$. Statistically, the amount of lactic acid produced by lactulose fermentation was not different from dextrose fermentation $(p=0.3299$; Figure 4(b)). Between FOS and lactulose, similar to the results obtained from the Bifidobacterium group, these two species produced lower $\mathrm{pH}$ and higher \% lactic acid when lactulose was the carbohydrate source instead of FOS $(p<0.0001$; Figure 4).

One of the key criteria for prebiotics is that they are selectively fermented by beneficial bacteria. In other words, for lactulose to be considered an effective

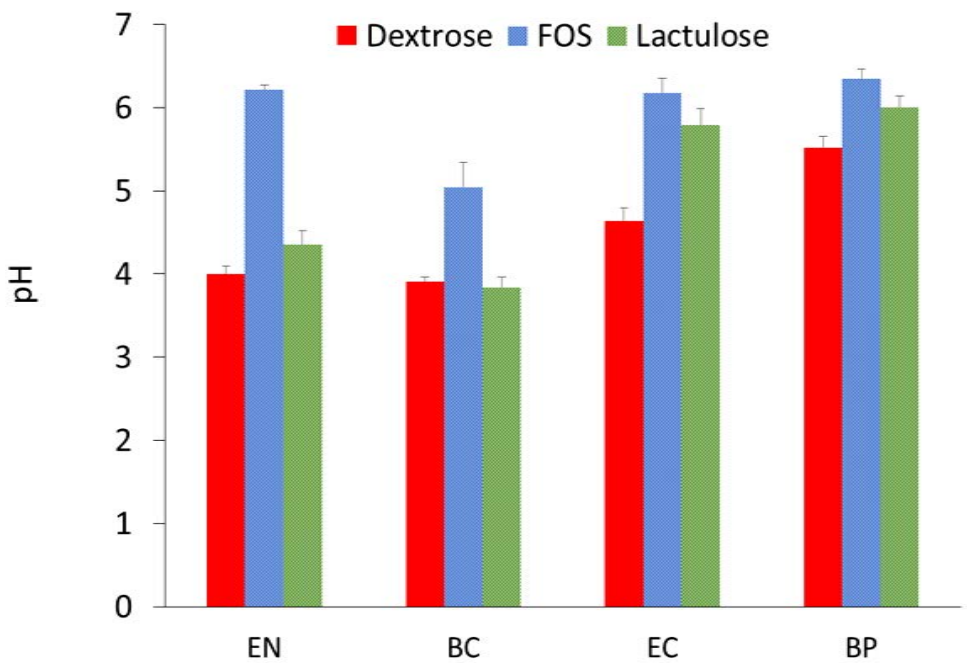

(a)

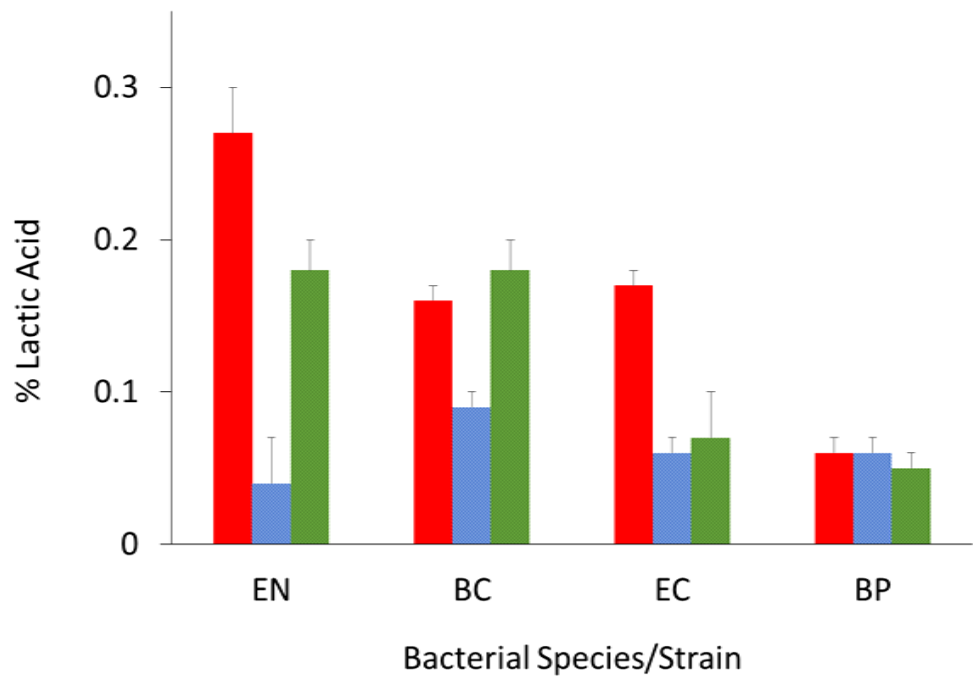

(b)

Figure 4. Mean \pm SE of $\mathrm{pH}$ (a) and \% lactic acid (b) of Escherichia and Bacillus spp. after anaerobic incubation for $24 \mathrm{hrs}$ at $37^{\circ} \mathrm{C}$ in medium containing dextrose, FOS, or lactulose as the sole carbohydrate source. Error bars indicate standard error of mean. $\mathrm{EN}=$ probiotic $E$. coli Nissle $1917, \mathrm{BC}=$ probiotic $B$. coagulans, $\mathrm{EC}=$ non-probiotic $E$. coli, $\mathrm{BP}=$ non-probiotic $B$. pumilus. 
prebiotic, probiotic strains should be able to better utilize lactulose when compared to non-probiotic strains. All probiotic strains in this study (Lactobacillus spp., Bifidobacterium spp., ECN, B. coagulans) yielded lower $\mathrm{pH}(p<0.0001)$ and higher \% lactic acid $(p<0.0001)$ when given lactulose (or FOS, so far as prebiotic potential is concerned) in comparison to the non-probiotic E. coli and B. pumilus (Figure 4). These results signify the prebiotic potential of lactulose.

\subsection{Antioxidant Activity}

Mild statistically difference was observed in TEAC levels among the different carbohydrate sources ( $p=0.0409$, Figure 5 ). When all strains were considered collectively, FOS as the sole or main carbohydrate source resulted in $0.32 \pm 0.14$ $\mathrm{mM}$ higher TEAC than dextrose. Further examination specifically on the Lactobacillus spp. showed that dextrose or lactulose fermentation did not produce significantly different TEAC levels $(p=0.0958)$; however the TEAC from FOS fermentation was significantly higher compared to dextrose $(p=0.0447)$. The Bifidobacterium spp. also produced a significantly higher TEAC when supplemented with FOS compared to lactulose $(p=0.0002)$. Lactulose fermentation of probiotic ECN and B. coagulans produced higher TEAC than dextrose or FOS fermentation but the difference was not statistically different $(p=0.2599)$. The non-probiotic group showed no differences in TEAC levels regardless of the carbohydrate sources ( $p=0.8816$, data not shown).

Amongst their many putative health benefits, probiotics may neutralize reactive oxygen species (ROS) in the host and repress oxidative stress [45] [46]. Madhu et al. (2012) investigated the antioxidant effect of adding 1\% (w/v) FOS to the starter cultures, Streptococcus thermophilus ATCC 19258 and L. delbrueckii ssp. bulgaricus CFR2028, supplemented with either Lactobacillus plantarum CFR2194 or Lactobacillus fermentum CFR2192 in yogurt samples. The total

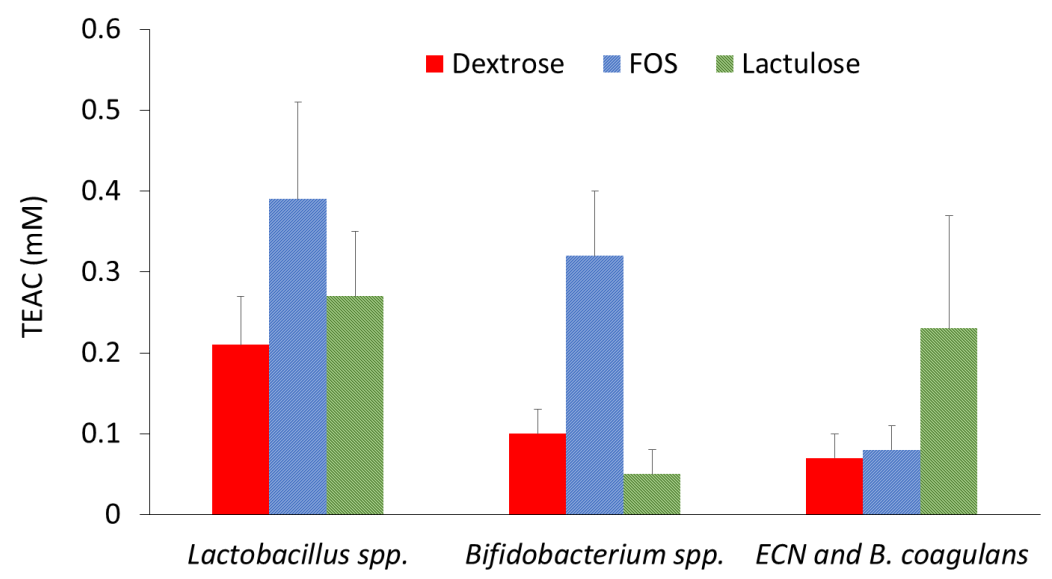

Figure 5. Mean \pm SE of Trolox equivalent antioxidant capacity (TEAC, $\mathrm{mM}$ ) of probiotics after anaerobic incubation for $24 \mathrm{hrs}$ at $37^{\circ} \mathrm{C}$ in medium containing dextrose, FOS, or lactulose as the sole or primary carbohydrate source. Error bars indicate standard error of mean. Each of the Lactobacillus spp. and Bifidobacterium spp. groups included four species/strains. E. coli Nissle (ECN) and B. coagulans represented non-lactic probiotics. 
antioxidant potential measured via ferric reducing ability (FRAP) assay showed that the synbiotic yogurt samples were able to scavenge $85 \%$ and $82 \%$ free radicals, when they contained L. plantarum and L. fermentum, respectively; compared to the $72 \%$ from the control samples containing the starter cultures only [47]. Our Lactobacillus strains performed comparably in their ability to produce higher TEAC levels with FOS supplementation than with dextrose $(p=0.0447)$.

Our results also show that the antioxidant activity is highly strain-dependent $(p=0.0026)$. Between the two $L$. casei strains, overnight incubation of ATCC 334 with FOS produced $0.74 \pm 0.29 \mathrm{mM}$ TEAC, whereas LcS produced $0.07 \pm$ $0.05 \mathrm{mM}$. Virtanen et al. (2007) showed that a combination of Leuconostoc cremoris B, Lactococcus lactis, and L. acidophilus in fermented milk resulted in higher radical scavenging activity than in milk fermented with a single bacterial strain [48], suggesting that a cocktail of carefully chosen probiotic strains might be the best approach to maximize the antioxidant activity of consumer products.

\section{Conclusion}

The goal of the present study was to compare FOS and lactulose in regard to their effects on the viability and fermentative activity of common probiotic bacteria. Different probiotic strains were tested, along with two non-probiotic strains for additional comparison. Similar to FOS, lactulose supported the growth and fermentative activity of probiotics. In case of Bifidobacterium spp., lactulose appeared to stimulate even higher acid production than FOS in anaerobic condition. Food product developers might consider lactulose as the choice of prebiotics when formulating consumer products containing bifidobacteria. Antioxidant seemed to be strain dependent, and while FOS appeared to be more effective than lactulose in stimulating the antioxidant activity of bifidobacteria, further investigations on combinations of probiotics and whether lactulose could be an effective prebiotic to support the activities of probiotic mixtures are warranted.

\section{Acknowledgements}

Partial funding for this project has been provided by the California State University Agricultural Research Institute (ARI).

\section{References}

[1] Ley, R.E., Hamady, M., Lozupone, C., Turnbaugh, P.J., Ramey, R.R., Bircher, J.S., Schlegel, M.L., Tucker, T.A., Schrenzel, M.D. and Knight, R. (2008) Evolution of Mammals and Their Gut Microbes. Science, 320, 1647-1651. https://doi.org/10.1126/science.1155725

[2] Ishibashi, N., Yaeshima, T. and Hayasawa, H. (1997) Bifidobacteria: Their Significance in Human Intestinal Health. Malaysian Journal of Nutrition, 3, 149-159.

[3] Maynard, C.L., Elson, C.O., Hatton, R.D. and Weaver, C.T. (2012) Reciprocal Interactions of the Intestinal Microbiota and Immune System. Nature, 489, 231-241. https://doi.org/10.1038/nature11551 
[4] Gibson, G.R., Scott, K.P., Rastall, R.A., Tuohy, K.M., Hotchkiss, A., Dubert-Ferrandon, A., Gareau, M., Murphy, E.F., Saulnier, D., Loh, G. and Macfarlane, S. (2010) Dietary Prebiotics: Current Status and New Definition. Food Science and Technology Bulletin: Functional Foods, 7, 1-19. https://doi.org/10.1616/1476-2137.15880

[5] Jeurink, P.V., van Esch, B.C., Rijnierse, A., Garssen, J. and Knippels, L.M. (2013) Mechanisms Underlying Immune Effects of Dietary Oligosaccharides. The American Journal of Clinical Nutrition, 98, 572S-577S.

https://doi.org/10.3945/ajcn.112.038596

[6] Spiegel, J.E., Rose, R., Karabell, P., Frankos, V.H. and Schmitt, D.F. (1994) Safety and Benefits of Fructooligosaccharides as Food Ingredients. Food Technology, 48, 85-89.

[7] Kato, K., Mizuno, S., Umesaki, Y., Ishii, Y., Sugitani, M., Imaoka, A., Otsuka, M., Hasunuma, O., Kurihara, R., Iwasaki, A. and Arakawa, Y. (2004) Randomized Placebo-Controlled Trial Assessing the Effect of Bifidobacteria-Fermented Milk on Active Ulcerative Colitis. Alimentary Pharmacology \& Therapeutics, 20, 1133-1141. https://doi.org/10.1111/j.1365-2036.2004.02268.x

[8] Ulluwishewa, D., Anderson, R.C., McNabb, W.C., Moughan, P.J., Wells, J.M. and Roy, N.C. (2011) Regulation of Tight Junction Permeability by Intestinal Bacteria and Dietary Components. The Journal of Nutrition, 141, 769-776.

https://doi.org/10.3945/jn.110.135657

[9] Zhang, F., Yung, K.K.L., Chung, S.S.M. and Yeung, C.K. (2017) Supplementation of Fructooligosaccharide Mildly Improves the Iron Status of Anemic Rats Fed a Low-Iron Diet. Food and Nutrition Sciences, 8, 294-304. https://doi.org/10.4236/fns.2017.82019

[10] Ito, M., Sawada, H., Ohishi, K., Yoshida, Y., Yokoi, W., Watanabe, T. and Yokokura, T. (2001) Suppressive Effects of Bifidobacteria on Lipid Peroxidation in the Colonic Mucosa of Iron-Overloaded Mice. Journal of Dairy Science, 84, 1583-1589. https://doi.org/10.3168/jds.S0022-0302(01)74591-2

[11] Şengül, N., Işık, S., Aslım, B., Uçar, G. and Demirbağ, A.E. (2011) The Effect of Exopolysaccharide-Producing Probiotic Strains on Gut Oxidative Damage in Experimental Colitis. Digestive Diseases and Sciences, 56, 707-714. https://doi.org/10.1007/s10620-010-1362-7

[12] Federico, A., Morgillo, F., Tuccillo, C., Ciardiello, F. and Loguercio, C. (2007) Chronic Inflammation and Oxidative Stress in Human Carcinogenesis. International Journal of Cancer, 121, 2381-2386. https://doi.org/10.1002/ijc.23192

[13] Fox, P.F. and McSweeney, P.L.H. (1998) Dairy Chemistry and Biochemistry.

[14] Fadden, K. and Owen, R.W. (1992) Faecal Steroids and Colorectal Cancer: The Effect of Lactulose on Faecal Bacterial Metabolism in a Continuous Culture Model of the Large Intestine. European Journal of Cancer Prevention, 1, 113-128. https://doi.org/10.1097/00008469-199202000-00004

[15] Nagendra, R. and Rao, S.V. (1992) Effect of Incorporation of Lactulose in Infant Formulas on the Intestinal Bifidobacteria! Flora in Rats. International Journal of Food Sciences and Nutrition, 43, 169-173. https://doi.org/10.3109/09637489209028369

[16] Terada, A., Hara, H., Kataoka, M. and Mitsuoka, T. (1992) Effect of Lactulose on the Composition and Metabolic Activity of the Human Faecal Flora. Microbial Ecology in Health and Disease, 5, 43-50. https://doi.org/10.3109/08910609209141303

[17] Crittenden, R.G. and Playne, M.J. (1996) Production, Properties and Applications of 
Food-Grade Oligosaccharides. Trends in Food Science \& Technology, 7, 353-361. https://doi.org/10.1016/S0924-2244(96)10038-8

[18] Playne, M.J. and Crittenden, R.G. (2009) Galacto-Oligosaccharides and Other Products Derived from Lactose. Advanced Dairy Chemistry, 3, 121-202.

[19] Gorski, D. (1997) Ingredient Forecast Product Development for World Markets. Dairy Foods, 98, 60-62.

[20] Roberfroid, M.B., Van Loo, J.A. and Gibson, G.R. (1998) The Bifidogenic Nature of Chicory Inulin and Its Hydrolysis Products. The Journal of Nutrition, 128, 11-19. https://doi.org/10.1093/jn/128.1.11

[21] Rao, V.A. (2001) The Prebiotic Properties of Oligofructose at Low Intake Levels. Nutrition Research, 21, 843-848. https://doi.org/10.1016/S0271-5317(01)00284-6

[22] Duar, R.M., Ang, P.T., Hoffman, M., Wehling, R., Hutkins, R. and Schlegel, V. (2015) Processing Effects on Four Prebiotic Carbohydrates Supplemented in an Extruded Cereal and a Low pH Drink. Cogent Food \& Agriculture, 1, Article ID: 1013782. https://doi.org/10.1080/23311932.2015.1013782

[23] Vega, R. and Zuniga-Hansen, M.E. (2015) The Effect of Processing Conditions on the Stability of Fructooligosaccharides in Acidic Food Products. Food Chemistry, 173, 784-789. https://doi.org/10.1016/j.foodchem.2014.10.119

[24] Ukena, S.N., Singh, A., Dringenberg, U., Engelhardt, R., Seidler, U., Hansen, W., Bleich, A., Bruder, D., Franzke, A. and Rogler, G. (2007) Probiotic Escherichia coli Nissle 1917 Inhibits Leaky Gut by Enhancing Mucosal Integrity. PLoS ONE, 2, e1308. https://doi.org/10.1371/journal.pone.0001308

[25] Henker, J., Laass, M., Blokhin, B.M., Bolbot, Y.K., Maydannik, V.G., Elze, M., Wolff, C. and Schulze, J. (2007) The Probiotic Escherichia coli Strain Nissle 1917 $(\mathrm{EcN})$ Stops Acute Diarrhoea in Infants and Toddlers. European Journal of Pediatrics, 166, 311-318. https://doi.org/10.1007/s00431-007-0419-x

[26] Watson, D., O’Connell Motherway, M., Schoterman, M.H.C., Neerven, R.J., Nauta, A. and Sinderen, D. (2013) Selective Carbohydrate Utilization by Lactobacilli and Bifidobacteria. Journal of Applied Microbiology, 114, 1132-1146. https://doi.org/10.1111/jam.12105

[27] Kaplan, H. and Hutkins, R.W. (2000) Fermentation of Fructooligosaccharides by Lactic Acid Bacteria and Bifidobacteria. Applied and Environmental Microbiology, 66, 2682-2684. https://doi.org/10.1128/AEM.66.6.2682-2684.2000

[28] Shigwedha, N., Hiwilepo-Van Hal, P., Jia, L., Sichel, L. and Zhang, S. (2016) Prebiotics: Metabolism and Symbiotic Synergy with Probiotics in Promoting Health. In: Rao, V. and Rao, L.G., Eds., Probiotics and Prebiotics in Human Nutrition and Health, InTech, London, Chapter 3.

[29] De Vries, W. and Stouthamer, A.H. (1969) Factors Determining the Degree of Anaerobiosis of Bifidobacterium Strains. Archivfür Mikrobiologie, 65, 275-287.

[30] Scaldaferri, F., Gerardi, V., Lopetuso, L.R., Del Zompo, F., Mangiola, F., Boškoski, I., Bruno, G., Petito, V., Laterza, L., Cammarota, G. and Gaetani, E. (2013) Gut Microbial Flora, Prebiotics, and Probiotics in IBD: Their Current Usage and Utility. BioMed Research International, 2013, Article ID: 435268.

[31] Tuohy, K.M., Probert, H.M., Smejkal, C.W. and Gibson, G.R. (2003) Using Probiotics and Prebiotics to Improve Gut Health. Drug Discovery Today, 8, 692-700. https://doi.org/10.1016/S1359-6446(03)02746-6

[32] Zuccotti, G.V., Meneghin, F., Raimondi, C., Dilillo, D., Agostoni, C., Riva, E. and Giovannini, M. (2008) Probiotics in Clinical Practice: An Overview. Journal of In- 
ternational Medical Research, 36, 1A-53A.

https://doi.org/10.1177/14732300080360S101

[33] Hong, H.A. and Cutting, S.M. (2005) The Use of Bacterial Spore Formers as Probiotics. FEMS Microbiology Reviews, 29, 813-835.

https://doi.org/10.1016/j.femsre.2004.12.001

[34] Cutting, S.M. (2011) Bacillus Probiotics. Food Microbiology, 28, 214-220.

https://doi.org/10.1016/j.fm.2010.03.007

[35] Endres, J., Clewell, A., Jade, K., Farber, T., Hauswirth, J. and Schauss, A. (2009) Safety Assessment of a Proprietary Preparation of a Novel Probiotic, Bacillus coagulans, as a Food Ingredient. Food and Chemical Toxicology, 47, 1231-1238. https://doi.org/10.1016/j.fct.2009.02.018

[36] O’Sullivan, L., Murphy, B., McLoughlin, P., Duggan, P., Lawlor, P.G., Hughes, H. and Gardiner, G.E. (2010) Prebiotics from Marine Macroalgae for Human and Animal Health Applications. Marine Drugs, 8, 2038-2064.

https://doi.org/10.3390/md8072038

[37] Wang, X. and Gibson, G. (1993) Effects of the in Vitro Fermentation of Oligofructose and Inulin by Bacteria Growing in the Human Large Intestine. Journal of Applied Bacteriology, 75, 373-380. https://doi.org/10.1111/j.1365-2672.1993.tb02790.x

[38] Schouler, C., Taki, A., Chouikha, I., Moulin-Schouleur, M. and Gilot, P. (2009) A Genomic Island of an Extraintestinal Pathogenic Escherichia coli Strain Enables the Metabolism of Fructooligosaccharides, Which Improves Intestinal Colonization. Journal of Bacteriology, 191, 388-393. https://doi.org/10.1128/JB.01052-08

[39] Ziemer, C.J. and Gibson, G.R. (1998) An Overview of Probiotics, Prebiotics and Synbiotics in the Functional Food Concept: Perspectives and Future Strategies. International Dairy Journal, 8, 473-479. https://doi.org/10.1016/S0958-6946(98)00071-5

[40] Fanaro, S., Chierici, R., Guerrini, P. and Vigi, V. (2003) Intestinal Microflora in Early Infancy: Composition and Development. Acta Paediatrica, 92, 48-55.

[41] Lievin, V., Peiffer, I., Hudault, S., Rochat, F., Brassart, D., Neeser, J.R. and Servin, A.L. (2000) Bifidobacterium Strains from Resident Infant Human Gastrointestinal Microflora Exert Antimicrobial Activity. Gut, 47, 646-652.

https://doi.org/10.1136/gut.47.5.646

[42] Rossi, M., Corradini, C., Amaretti, A., Nicolini, M., Pompei, A., Zanoni, S. and Matteuzzi, D. (2005) Fermentation of Fructooligosaccharides and Inulin by Bifidobacteria: A Comparative Study of Pure and Fecal Cultures. Applied and Environmental Microbiology, 71, 6150-6158. https://doi.org/10.1128/AEM.71.10.6150-6158.2005

[43] Valdés-Varela, L., Ruas-Madiedo, P. and Gueimonde, M. (2017) In Vitro Fermentation of Different Fructo-Oligosaccharides by Bifidobacterium Strains for the Selection of Synbiotic Combinations. International Journal of Food Microbiology, 242, 19-23. https://doi.org/10.1016/j.ijfoodmicro.2016.11.011

[44] Langlands, S.J., Hopkins, M.J., Coleman, N. and Cummings, J.H. (2004) Prebiotic Carbohydrates Modify the Mucosa Associated Microflora of the Human Large Bowel. Gut, 53, 1610-1616. https://doi.org/10.1136/gut.2003.037580

[45] Martarelli, D., Verdenelli, M.C., Scuri, S., Cocchioni, M., Silvi, S., Cecchini, C. and Pompei, P. (2011) Effect of a Probiotic Intake on Oxidant and Antioxidant Parameters in Plasma of Athletes during Intense Exercise Training. Current Microbiology, 62, 1689-1696. https://doi.org/10.1007/s00284-011-9915-3 
[46] Ejtahed, H.S., Mohtadi-Nia, J., Homayouni-Rad, A., Niafar, M., Asghari-Jafarabadi, M. and Mofid, V. (2012) Probiotic Yogurt Improves Antioxidant Status in Type 2 Diabetic Patients. Nutrition, 28, 539-543. https://doi.org/10.1016/j.nut.2011.08.013

[47] Madhu, A.N., Amrutha, N. and Prapulla, S.G. (2012) Characterization and Antioxidant Property of Probiotic and Synbiotic Yogurts. Probiotics and Antimicrobial Proteins, 4, 90-97. https://doi.org/10.1007/s12602-012-9099-6

[48] Virtanen, T., Pihlanto, A., Akkanen, S. and Korhonen, H. (2007) Development of Antioxidant Activity in Milk Whey during Fermentation with Lactic Acid Bacteria. Journal of Applied Microbiology, 102, 106-115. https://doi.org/10.1111/j.1365-2672.2006.03072.x 